\title{
IDENTITIES ARISING FROM GAUSS SUMS FOR SYMPLECTIC AND ORTHOGONAL GROUPS
}

\author{
Hi-Joon Chae and Dae San Kim
}

\begin{abstract}
We express Gauss sums for symplectic and orthogonal groups over finite fields as averages of exponential sums over certain maximal tori. Together with our previous results, we obtain some interesting identities involving various classical Gauss and Kloosterman sums.
\end{abstract}

\section{Introduction}

In a series of papers $[7,8,9,10,11,12,15]$, the second author considered Gauss sums for various finite classical groups, which are exponential sums of the form

$$
\sum_{g \in G^{F}} \chi(\operatorname{det} g) \lambda\left((\operatorname{tr} g)^{r}\right)
$$

where $G$ is a classical group over a finite field, $F$ is the Frobenius map, $G^{F}$ denotes the set of fixed points of $G$ under $F$, and $\chi$ (resp. $\lambda$ ) is a multiplicative (resp. nontrivial additive) character of the finite field. He obtained explicit expressions of these sums in terms of classical Gauss and Kloosterman sums and proved some applications. On the other hand, we proved the next formula in [3, Theorem 3.1], which follows from the representation theory of finite reductive groups: let $\psi$ be a complex valued class function on $G\left(\overline{\mathbb{F}}_{q}\right)$ whose value depend only on semisimple part of each element. Then we have

$$
\sum_{g \in G^{F}} \psi(g)=\frac{\left|G^{F}\right|}{|W|} \sum_{w \in W} \frac{1}{\left|T^{w} F\right|} \sum_{a \in T w F} \psi(a),
$$

where $T$ is an $F$-stable maximal torus of $G, W=N(T) / T$ is the Weyl group of $G$ with respect to $T$, and ${ }_{w} F$ is the twisted Frobenius map of $T$ given by ${ }_{w} F(t)=w F(t) w^{-1}$.

In this paper, we apply the above formula to calculate the Gauss sums for $\mathrm{Sp}_{2 n}, \mathrm{GSp}_{2 n}, \mathrm{SO}_{2 n}^{ \pm}$, and $\mathrm{SO}_{2 n+1}$ (Theorems 3.1, 3.2, 3.5, 3.6, and 3.7). Combined with the second author's previous results cited above, these yield new

Received June 11, 2008.

2000 Mathematics Subject Classification. 55A19, 11T24, $20 \mathrm{G} 40$.

Key words and phrases. Gauss sums, exponential sums, symplectic groups, orthogonal groups. 
identities involving various classical Gauss and Kloosterman sums (Theorems $4.1,4.3$, and 4.5), which may be of interest in number theory and combinatorics.

Another interesting consequence is the complete relations among Gauss sums for $\operatorname{Sp}(2 n, q), \mathrm{SO}(2 n+1, q), \mathrm{SO}^{+}(2 n, q)$, and $\mathrm{SO}^{-}(2 n, q)$. Namely, we have the following identities (Theorem 4.8$)$ :

$$
\begin{aligned}
\sum_{g \in \operatorname{SO}(2 n+1, q)} \lambda(\operatorname{tr} g) & =\lambda(1) \sum_{g \in \operatorname{Sp}(2 n, q)} \lambda(\operatorname{tr} g), \\
\sum_{g \in \mathrm{SO}^{+}(2 n, q)} \lambda(\operatorname{tr} g) & =q^{-n} \sum_{g \in \operatorname{Sp}(2 n, q)} \lambda(\operatorname{tr} g), \\
\sum_{g \in \mathrm{SO}^{-}(2 n, q)} \lambda(\operatorname{tr} g) & =-q^{-n} \sum_{g \in \operatorname{Sp}(2 n, q)} \lambda(\operatorname{tr} g) .
\end{aligned}
$$

The first of these was noted in $[11,(5.12)]$. However, as we confessed at that time, we didn't have any philosophical reason that this should be so. Now, that is an easy consequence of the main formula (1). The second identity was observed in [15], while the last one was obtained from the old and new expressions of Gauss sums for $\mathrm{SO}^{+}(2 n, q)$ and $\mathrm{SO}^{-}(2 n, q)$. This last identity would have been found earlier if we pushed ourselves a little further in getting an expression of Gauss sums for $\mathrm{SO}^{-}(2 n, q)$, as in Proposition 4.9.

\section{Preliminaries and notations}

One is referred to [2] and [17] for some elementary facts below. Let $\mathbb{F}_{q}$ be the finite field with $q$ elements. Then, for each $m \in \mathbb{Z}_{>0}, \mathbb{F}_{q^{m}}$ denotes the extension field of degree $m$ of $\mathbb{F}_{q}$ in a fixed algebraic closure $\overline{\mathbb{F}}_{q}$ of $\mathbb{F}_{q}$. For each $m \in \mathbb{Z}_{>0}, C_{q^{m}}$ denotes the kernel of the norm map $N_{\mathbb{F}_{q^{2 m}} / \mathbb{F}_{q^{m}}}: \mathbb{F}_{q^{2 m}}^{\times} \rightarrow \mathbb{F}_{q^{m}}^{\times}$, so that

$$
C_{q^{m}}=\left\{\alpha \in \mathbb{F}_{q^{2 m}} \mid \alpha^{q^{m}+1}=1\right\} .
$$

Let $\lambda$ be a nontrivial additive character of $\mathbb{F}_{q}, \chi$ a multiplicative character of $\mathbb{F}_{q}$, and let $\tilde{\chi}$ be a multiplicative character of $\mathbb{F}_{q^{2}}$. For each $m \in \mathbb{Z}_{>0}$, we put

$$
\lambda^{(m)}=\lambda \circ \operatorname{tr}_{\mathbb{F}_{q} m / \mathbb{F}_{q}}, \chi^{(m)}=\chi \circ N_{\mathbb{F}_{q} m / \mathbb{F}_{q}}, \tilde{\chi}^{(2 m)}=\tilde{\chi} \circ N_{\mathbb{F}_{q^{2 m}} / \mathbb{F}_{q^{2}}} .
$$

To simplify notations, the lifting $\lambda^{(2)}$ of $\lambda$ to $\mathbb{F}_{q^{2}}$ will be denoted by $\lambda^{\prime}$. For $m \in \mathbb{Z}_{>0}$ and $\gamma \in \mathbb{F}_{q}^{\times}$, we put

$$
\begin{aligned}
K\left(\lambda^{(m)} ; \gamma\right) & =\sum_{\alpha \in \mathbb{F}_{q^{m}}^{\times}} \lambda^{(m)}\left(\alpha+\gamma \alpha^{-1}\right), \\
K\left(\lambda^{(m)}\right) & =K\left(\lambda^{(m)} ; 1\right)=\sum_{\alpha \in \mathbb{F}_{q^{m}}^{\times}} \lambda^{(m)}\left(\alpha+\alpha^{-1}\right),
\end{aligned}
$$




$$
\begin{aligned}
& K\left(\lambda^{(2 m)}, \tilde{\chi}^{(2 m)}\right)=\sum_{\alpha \in \mathbb{F}_{q^{2 m}}^{\times}} \tilde{\chi}^{(2 m)}(\alpha) \lambda^{(2 m)}\left(\alpha+\alpha^{-1}\right), \\
& J\left(\lambda^{(m)} ; \gamma\right)=\sum_{\alpha \in N_{\mathbb{F}_{q} 2 m / \mathbb{F} q}^{-1}(\gamma)} \lambda^{(m)}\left(\alpha+\gamma \alpha^{-1}\right) \\
& =\sum_{\alpha \in N_{\mathbb{F}_{q}^{2 m} / \mathbb{F}_{q} m}^{-1}(\gamma)} \lambda^{(2 m)}(\alpha), \\
& J\left(\lambda^{(m)}\right)=J\left(\lambda^{(m)} ; 1\right)=\sum_{\alpha \in C_{q} m} \lambda^{(m)}\left(\alpha+\alpha^{-1}\right) \\
& =\sum_{\alpha \in C_{q} m} \lambda^{(2 m)}(\alpha), \\
& J\left(\lambda^{(m)}, \widetilde{\chi}^{(2 m)}\right)=\sum_{\alpha \in C_{q^{m}}} \tilde{\chi}^{(2 m)}(\alpha) \lambda^{(m)}\left(\alpha+\alpha^{-1}\right) \\
& =\sum_{\alpha \in C_{q^{m}}} \tilde{\chi}^{(2 m)}(\alpha) \lambda^{(2 m)}(\alpha),
\end{aligned}
$$

$K(\lambda ; \gamma), K\left(\lambda^{\prime}, \widetilde{\chi}\right), J(\lambda ; \gamma)$, and $J(\lambda, \widetilde{\chi})$ are respectively called a Kloosterman sum, a twisted Kloosterman sum, a unitary Kloosterman sum, and a twisted unitary Kloosterman sum. The ones in (4), (6), (7), (9) are liftings of those classical exponential sums. In addition, we need the Gauss sum $G(\chi, \lambda)$ and the hyperkloosterman sum $K_{m}(\lambda)$ which are respectively defined by

$$
\begin{gathered}
G(\chi, \lambda)=\sum_{\alpha \in \mathbb{F}_{q}^{\times}} \chi(\alpha) \lambda(\alpha), \\
K_{m}(\lambda ; \gamma)=\sum_{\alpha_{1}, \ldots, \alpha_{m} \in \mathbb{F}_{q}^{\times}} \lambda\left(\alpha_{1}+\cdots+\alpha_{m}+\gamma \alpha_{1}^{-1} \cdots \alpha_{m}^{-1}\right), \\
K_{m}(\lambda)=K_{m}(\lambda ; 1)=\sum_{\alpha_{1}, \ldots, \alpha_{m} \in \mathbb{F}_{q}^{\times}} \lambda\left(\alpha_{1}+\cdots+\alpha_{m}+\alpha_{1}^{-1} \cdots \alpha_{m}^{-1}\right) .
\end{gathered}
$$

For $\alpha \in \overline{\mathbb{F}}_{q}^{\times}, m \in \mathbb{Z}_{>0}$, we will need the following diagonal matrices:

$$
\begin{aligned}
& t_{m}(\alpha)=\operatorname{diag}\left(\alpha, \alpha^{q}, \ldots, \alpha^{q^{m-1}}\right), \\
& t_{m}^{\prime}(\alpha)=\operatorname{diag}\left(\alpha, \alpha^{-q}, \ldots, \alpha^{(-q)^{m-1}}\right) .
\end{aligned}
$$

We now recall some basic terminologies and notations about partitions from [18]. A partition of a positive integer $n$ is a finite nondecreasing sequence $\mu_{1}, \ldots, \mu_{s}$ of positive integers with $\sum_{i=1}^{s} \mu_{i}=n$. The partition $\left(\mu_{1}, \ldots, \mu_{s}\right)$ is denoted by $\mu$, and we write

$$
\mu \vdash n \quad \text { if } \mu \text { is a partition of } n \text {. }
$$


The $\mu_{i}$ 's are the parts of $\mu$, and

$$
\begin{aligned}
l(\mu) & =\text { the number of parts of } \mu, \\
|\mu| & =\text { the sum of parts of } \mu .
\end{aligned}
$$

The multiplicity of $i$ in $\mu \vdash n$ is

$$
m_{i}=m_{i}(\mu)=\left|\left\{j \mid 1 \leqslant j \leqslant s, \mu_{j}=i\right\}\right|,
$$

in which case we write

$$
\mu=\left(1^{m_{1}} 2^{m_{2}} \cdots n^{m_{n}}\right) .
$$

So $\sum_{i=1}^{n} i m_{i}=n$. The set of all partitions of $n$ is denoted by $\mathcal{P}_{n}\left(n \in \mathbb{Z}_{\geqslant 0}\right)$, and the set of all partitions $\bigcup_{n \in \mathbb{Z}} \mathcal{P}_{n}$ by $\mathcal{P}$. Here $\mathcal{P}_{0}$ consists of a single element, the 'empty partition of 0 ', which we denote by $\mathbf{0}$. If $\mu=\left(1^{m_{1}} 2^{m_{2}} \cdots n^{m_{n}}\right)$ is a partition of $n$, we write

$$
z_{\mu}=1^{m_{1}} 2^{m_{2}} \cdots n^{m_{n}} m_{1} ! m_{2} ! \cdots m_{n} !
$$

For $\mu=\mathbf{0} \in \mathcal{P}_{0}$, we agree that

$$
l(\mu)=0,|\mu|=0, z_{\mu}=1 .
$$

For integers $n, r$ with $0 \leqslant r \leqslant n$, the $q$-binomial coefficients are defined by

$$
\left[\begin{array}{l}
n \\
r
\end{array}\right]_{q}=\prod_{j=0}^{r-1} \frac{q^{n-j}-1}{q^{r-j}-1} .
$$

$[y]$ denotes the greatest integer $\leqslant y$ for a real number $y$.

\section{Calculation of Gauss sums}

In this section we apply the formula (1) to calculate the Gauss sums $\sum \lambda(\operatorname{tr} g)$ for symplectic and orthogonal groups. In doing so, since the summand of the summation over $w \in W$ in the right hand side of (1) depends only on the $F$-conjugacy class $\left\{v^{-1} w F(v) \mid v \in W\right\}$ of $w$ in $W$ (cf. the proof of [3, Theorem 3.1]), we will sum over the $F$-conjugacy classes in $W$ with summands multiplied by their orders.

\subsection{Symplectic groups}

Let $G=\operatorname{Sp}\left(2 n, \overline{\mathbb{F}}_{q}\right)=\left\{\left.g \in \mathrm{GL}\left(2 n, \overline{\mathbb{F}}_{q}\right)\right|^{t} g \widetilde{J} g=\widetilde{J}\right\}$, with $\widetilde{J}=\left[\begin{array}{cc}0 & 1_{n} \\ -1_{n} & 0\end{array}\right] . F$ is the standard Frobenius map $\operatorname{Fr}_{q}$ on $G$, and $G^{F}=\operatorname{Sp}(2 n, q)$, with

$$
|\operatorname{Sp}(2 n, q)|=q^{n^{2}} \prod_{j=1}^{n}\left(q^{2 j}-1\right) .
$$

$T=\{$ diagonal matrices in $G\}=\left\{\operatorname{diag}\left(\alpha_{1}, \ldots, \alpha_{n}, \alpha_{1}^{-1}, \ldots, \alpha_{n}^{-1}\right)\right\}$ is an $F$ stable maximal torus of $G$ which is split over $\mathbb{F}_{q} . W=N(T) / T \cong(\mathbb{Z} / 2 \mathbb{Z})^{n} \rtimes S_{n}$ is the Weyl group with respect to $T$ and $F$-conjugacy classes in $W$ are just 
conjugacy classes in $W$, which are in bijective correspondence with the set of double partitions of $n$

$$
\mathcal{P}_{n}^{(2)}=\{(\mu, \nu)|\mu, \nu \in \mathcal{P},| \mu|+| \nu \mid=n\} \quad(\text { cf. }(17)) .
$$

Here in $\mathcal{P}_{n}^{(2)}$ the special pairs $(\mathbf{0}, \nu)$ with $\nu \in \mathcal{P}_{n}$ or $(\mu, \mathbf{0})$ with $\mu \in \mathcal{P}_{n}$ are included (cf. (19)).

If the conjugacy class $\operatorname{conj}(w)$ of $w$ in $W$ corresponds to the double partition $(\mu, \nu)=\left(\left(\mu_{1}, \ldots, \mu_{s}\right),\left(\nu_{1}, \ldots, \nu_{t}\right)\right) \in \mathcal{P}_{n}^{(2)}$, then, in the notation of (13),

$$
\begin{gathered}
T^{w F}=\left\{\operatorname { d i a g } \left(t_{\mu_{1}}\left(\alpha_{1}\right), \ldots, t_{\mu_{s}}\left(\alpha_{s}\right), t_{\nu_{1}}\left(\beta_{1}\right), \ldots, t_{\nu_{t}}\left(\beta_{t}\right),\right.\right. \\
\left.t_{\mu_{1}}\left(\alpha_{1}^{-1}\right), \ldots, t_{\mu_{s}}\left(\alpha_{s}^{-1},\right), t_{\nu_{1}}\left(\beta_{1}^{-1}\right), \ldots, t_{\nu_{t}}\left(\beta_{t}^{-1}\right)\right) \mid \\
\left.\alpha_{1} \in \mathbb{F}_{q^{\mu_{1}}}^{\times}, \ldots, \alpha_{s} \in \mathbb{F}_{q^{\mu_{s}}}^{\times}, \beta_{1} \in C_{q^{\nu_{1}}}, \ldots, \beta_{t} \in C_{q^{\nu_{t}}}\right\} \\
\cong \mathbb{F}_{q^{\mu_{1}}}^{\times} \times \cdots \times \mathbb{F}_{q^{\mu_{s}}}^{\times} \times C_{q^{\nu_{1}}} \times \cdots \times C_{q^{\nu_{t}}}(\mathrm{cf} .(2)),
\end{gathered}
$$

$$
|\operatorname{conj}(w)|=\frac{|W|}{2^{l(\mu)+l(\nu)} z_{\mu} z_{\nu}}=\frac{2^{n} n !}{2^{l(\mu)+l(\nu)} z_{\mu} z_{\nu}} .
$$

Also, we have

$$
\begin{aligned}
\sum_{a \in T w F} \lambda(\operatorname{tr} a)= & \sum_{\alpha_{1} \in \mathbb{F}_{q^{\mu_{1}}}^{\times}} \lambda^{\left(\mu_{1}\right)}\left(\alpha_{1}+\alpha_{1}^{-1}\right) \cdots \sum_{\alpha_{s} \in \mathbb{F}_{q^{\mu_{s}}}^{\times}} \lambda^{\left(\mu_{s}\right)}\left(\alpha_{s}+\alpha_{s}^{-1}\right) \\
& \times \sum_{\beta_{1} \in C_{q^{\nu_{1}}}} \lambda^{\left(\nu_{1}\right)}\left(\beta_{1}+\beta_{1}^{-1}\right) \cdots \sum_{\beta_{t} \in C_{q^{\nu t}}} \lambda^{\left(\nu_{t}\right)}\left(\beta_{t}+\beta_{t}^{-1}\right) \\
= & K\left(\lambda^{\left(\mu_{1}\right)}\right) \cdots K\left(\lambda^{\left(\mu_{s}\right)}\right) J\left(\lambda^{\left(\nu_{1}\right)}\right) \cdots J\left(\lambda^{\left(\nu_{t}\right)}\right) \\
= & (-1)^{t} K\left(\lambda^{\left(\mu_{1}\right)}\right) \cdots K\left(\lambda^{\left(\mu_{s}\right)}\right) K\left(\lambda^{\left(\nu_{1}\right)}\right) \cdots K\left(\lambda^{\left(\nu_{t}\right)}\right)
\end{aligned}
$$

which follows from (5), (8), and the following fact which is a special case of [5, (7.2.5)]: for a nontrivial additive character $\lambda$ of $\mathbb{F}_{q}, \gamma \in \mathbb{F}_{q}^{\times}$, and $m \in \mathbb{Z}_{>0}$,

$$
J\left(\lambda^{(m)} ; \gamma\right)=-K\left(\lambda^{(m)} ; \gamma\right)
$$

The next result follows now from (1) and the above.

\section{Theorem 3.1.}

$$
\begin{aligned}
& \sum_{g \in \operatorname{Sp}(2 n, q)} \lambda(\operatorname{tr} g) \\
= & q^{n^{2}} \prod_{j=1}^{n}\left(q^{2 j}-1\right) \\
& \times \sum_{(\mu, \nu) \in \mathcal{P}_{n}^{(2)}} \frac{(-1)^{l(\nu)} K\left(\lambda^{\left(\mu_{1}\right)}\right) \cdots K\left(\lambda^{\left(\mu_{s}\right)}\right) K\left(\lambda^{\left(\nu_{1}\right)}\right) \cdots K\left(\lambda^{\left(\nu_{t}\right)}\right)}{2^{l(\mu)+l(\nu)} z_{\mu} z_{\nu}\left(q^{\mu_{1}}-1\right) \cdots\left(q^{\mu_{s}}-1\right)\left(q^{\nu_{1}}+1\right) \cdots\left(q^{\nu_{t}}+1\right)}
\end{aligned}
$$




\subsection{Symplectic similitude groups}

Let $G=\operatorname{GSp}\left(2 n, \overline{\mathbb{F}}_{q}\right)=\left\{\left.g \in \operatorname{GL}\left(2 n, \overline{\mathbb{F}}_{q}\right)\right|^{t} g \widetilde{J} g=\nu(g) \widetilde{J}\right.$ for some $\nu(g) \in$ $\left.\overline{\mathbb{F}}_{q}^{\times}\right\}$, with $\widetilde{J}=\left[\begin{array}{cc}0 & 1_{n} \\ -1_{n} & 0\end{array}\right] . F$ is the standard Frobenius map $\operatorname{Fr}_{q}$ on $G$, so that

$$
G^{F}=\operatorname{GSp}(2 n, q)=\left\{g \in \mathrm{GL}(2 n, q) \mid{ }^{t} g \widetilde{J} g=\nu(g) \widetilde{J} \text { for some } \nu(g) \in \mathbb{F}_{q}^{\times}\right\},
$$

with

$$
|\operatorname{GSp}(2 n, q)|=(q-1) q^{n^{2}} \prod_{j=1}^{n}\left(q^{2 j}-1\right) .
$$

$T=\{$ diagonal matrices in $G\}=\left\{\operatorname{diag}\left(\alpha_{1}, \ldots, \alpha_{n}, \gamma \alpha_{1}^{-1}, \ldots, \gamma \alpha_{n}^{-1}\right)\right\}$ is an $F$ stable maximal torus of $G$ which is split over $\mathbb{F}_{q} . W=N(T) / T \cong(\mathbb{Z} / 2 \mathbb{Z})^{n} \rtimes S_{n}$ is the Weyl group with respect to $T$, and $F$-conjugacy classes in $W$ are just conjugacy classes in $W$.

If $\operatorname{conj}(w)(w \in W)$ corresponds to $(\mu, \nu)=\left(\left(\mu_{1}, \ldots, \mu_{s}\right),\left(\nu_{1}, \ldots, \nu_{t}\right)\right) \in$ $\mathcal{P}_{n}^{(2)}$ (cf. (20)), then, in the notation of (13),

$$
\begin{gathered}
T^{w} F=\left\{\operatorname { d i a g } \left(t_{\mu_{1}}\left(\alpha_{1}\right), \ldots, t_{\mu_{s}}\left(\alpha_{s}\right), t_{\nu_{1}}\left(\beta_{1}\right), \ldots, t_{\nu_{t}}\left(\beta_{t}\right),\right.\right. \\
\left.\gamma t_{\mu_{1}}\left(\alpha_{1}^{-1}\right), \ldots, \gamma t_{\mu_{s}}\left(\alpha_{s}^{-1}\right), \gamma t_{\nu_{1}}\left(\beta_{1}^{-1}\right), \ldots, \gamma t_{\nu_{t}}\left(\beta_{t}^{-1}\right)\right) \mid \\
\gamma \in \mathbb{F}_{q}^{\times}, \alpha_{1} \in \mathbb{F}_{q^{\mu_{1}}}^{\times}, \ldots, \alpha_{s} \in \mathbb{F}_{q^{\mu_{s}}}^{\times}, \\
\left.\beta_{1} \in N_{\mathbb{F}_{q}^{2 \nu_{1}} / \mathbb{F}_{q}^{\nu_{1}}}^{-1}(\gamma), \ldots, \beta_{t} \in N_{\mathbb{F}_{q}^{2 \nu_{t}} / \mathbb{F}_{q}^{\nu_{t}}}^{-1}(\gamma)\right\} .
\end{gathered}
$$

Hence

$$
\begin{aligned}
& \sum_{a \in T w F} \chi(\operatorname{det} a) \lambda(\operatorname{tr} a) \\
= & \sum_{\gamma \in \mathbb{F}_{q}^{\times}} \chi^{n}(\gamma) K\left(\lambda^{\left(\mu_{1}\right)} ; \gamma\right) \cdots K\left(\lambda^{\left(\mu_{s}\right)} ; \gamma\right) J\left(\lambda^{\left(\nu_{1}\right)} ; \gamma\right) \cdots J\left(\lambda^{\left(\nu_{t}\right)} ; \gamma\right) \\
= & (-1)^{t} \sum_{\gamma \in \mathbb{F}_{q}^{\times}} \chi^{n}(\gamma) K\left(\lambda^{\left(\mu_{1}\right)} ; \gamma\right) \cdots K\left(\lambda^{\left(\mu_{s}\right)} ; \gamma\right) K\left(\lambda^{\left(\nu_{1}\right)} ; \gamma\right) \cdots K\left(\lambda^{\left(\nu_{t}\right)} ; \gamma\right)
\end{aligned}
$$

(cf. (4), (7), (23)).

The following theorem now follows from (1), (21) and the above.

Theorem 3.2.

$$
\begin{aligned}
& \sum_{g \in \operatorname{GSp}(2 n, q)} \chi(\operatorname{det} g) \lambda(\operatorname{tr} g) \\
= & q^{n^{2}} \prod_{j=1}^{n}\left(q^{2 j}-1\right) \\
& \times \sum_{(\mu, \nu) \in \mathcal{P}_{n}^{(2)}} \frac{(-1)^{l(\nu)} \sum_{\gamma \in \mathbb{F}_{q}^{\times}} \chi^{n}(\gamma) K\left(\lambda^{\left(\mu_{1}\right)} ; \gamma\right) \cdots K\left(\lambda^{\left(\mu_{s}\right)} ; \gamma\right) K\left(\lambda^{\left(\nu_{1}\right)} ; \gamma\right) \cdots K\left(\lambda^{\left(\nu_{t}\right)} ; \gamma\right)}{2^{l(\mu)+l(\nu)} z_{\mu} z_{\nu}\left(q^{\mu_{1}}-1\right) \cdots\left(q^{\mu_{s}}-1\right)\left(q^{\nu_{1}}+1\right) \cdots\left(q^{\nu_{t}}+1\right)}
\end{aligned}
$$


Remark 3.3. Let $\chi^{n}$ be trivial. Then the sum in the numerator of the summand of RHS of the above equation is

$$
\sum_{\gamma \in \mathbb{F}_{q}^{\times}} K\left(\lambda^{\left(\mu_{1}\right)} ; \gamma\right) \cdots K\left(\lambda^{\left(\mu_{s}\right)} ; \gamma\right) K\left(\lambda^{\left(\nu_{1}\right)} ; \gamma\right) \cdots K\left(\lambda^{\left(\nu_{t}\right)} ; \gamma\right)
$$

which, according to $[4$, Theorem 1$]$, is equal to

$$
\frac{q^{2}}{q-1}\left|A_{(\mu, \nu)}\right|-\frac{\left(q^{\mu_{1}}-1\right) \cdots\left(q^{\mu_{s}}-1\right)\left(q^{\nu_{1}}-1\right) \cdots\left(q^{\nu_{t}}-1\right)}{q-1}+2(-1)^{l(\mu)+l(\nu)-1}
$$

with

$$
\begin{aligned}
& A_{(\mu, \nu)}=\left\{\left(\alpha_{1}, \ldots, \alpha_{s}, \beta_{1}, \ldots, \beta_{t}\right) \in \mathbb{F}_{q^{\mu_{1}}}^{\times} \times \cdots \times \mathbb{F}_{q^{\mu_{s}}}^{\times} \times \mathbb{F}_{q^{\nu_{1}}}^{\times} \times \cdots \mathbb{F}_{q^{\nu t}}^{\times}\right. \\
& \sum_{i=1}^{s} \operatorname{tr}_{\mathbb{F}_{q} \mu_{i} / \mathbb{F}_{q}}\left(\alpha_{i}\right)+\sum_{j=1}^{t} \operatorname{tr}_{\mathbb{F}_{q} \nu_{j} / \mathbb{F}_{q}}\left(\beta_{j}\right) \\
& \left.=0=\sum_{i=1}^{s} \operatorname{tr}_{\mathbb{F}_{q} \mu_{i} / \mathbb{F}_{q}}\left(\alpha_{i}^{-1}\right)+\sum_{j=1}^{t} \operatorname{tr}_{\mathbb{F}_{q^{\nu}} / \mathbb{F}_{q}}\left(\beta_{j}^{-1}\right)\right\} .
\end{aligned}
$$

As $\left|A_{(\mu, \nu)}\right|$ is divisible by $q-1$, it is an integer, depending only on $(\mu, \nu)$.

\section{3. $\mathrm{SO}^{+}(2 n, q)$}

Let $J^{+}$be the $2 n \times 2 n$ matrix with $\left[\begin{array}{ll}0 & 1 \\ 1 & 0\end{array}\right]$ along the diagonal blocks and zeros elsewhere, and let

$$
G=\mathrm{SO}^{+}\left(2 n, \overline{\mathbb{F}}_{q}\right)=\left\{\left.g \in \mathrm{GL}\left(2 n, \overline{\mathbb{F}}_{q}\right)\right|^{t} g J^{+} g=J^{+}, \operatorname{det} g=1\right\} .
$$

If $F$ is the standard Frobenius map $\operatorname{Fr}_{q}$ on $G, G^{F}=\mathrm{SO}^{+}(2 n, q)$, with

$$
\left|\mathrm{SO}^{+}(2 n, q)\right|=q^{n^{2}-n}\left(q^{n}-1\right) \prod_{j=1}^{n-1}\left(q^{2 j}-1\right) .
$$

$T=\{$ diagonal matrices in $G\}$ is an $F$-stable maximal torus of $G$ which is split over $\mathbb{F}_{q}$. The Weyl group $W=N(T) / T$ with respect to $T$ is the subgroup of $W^{\prime}=(\mathbb{Z} / 2 \mathbb{Z})^{n} \rtimes S_{n}$ consisting of even signed permutations. A signed permutation $a \sigma \in W^{\prime}$, with $a=\left(a_{1}, \ldots, a_{n}\right) \in(\mathbb{Z} / 2 \mathbb{Z})^{n}=\{ \pm 1\}^{n}, \sigma \in S_{n}$, is called even (resp. odd) signed if $a_{1} \cdots a_{n}=1$ (resp. $a_{1} \cdots a_{n}=-1$ ).

$F$ acts trivially on $W$, and hence the $F$-conjugacy classes in $W$ coincide with conjugacy classes in $W$. Since $\left[W^{\prime}: W\right]=2$, the conjugacy class $\operatorname{conj}_{W^{\prime}}(w)$ of each $w \in W$ in $W^{\prime}$ is either a single conjugacy class in $W$ or the union of two conjugacy classes in $W$. More precisely, we have the following lemma.

Lemma 3.4. Let $W \subset W^{\prime}$ be as above. The conjugacy class conj ${ }_{W^{\prime}}(w)$ of $w \in$ $W$ in $W^{\prime}$ coincides with the conjugacy class $\operatorname{conj}_{W}(w)$ of $w$ in $W$, except when the double partition of $n$ corresponding to $\operatorname{conj}_{W^{\prime}}(w)$ is of the form $(\mu, \mathbf{0}) \in$ $\mathcal{P}_{n} \times \mathcal{P}_{0}$, with $\mu_{1}, \ldots, \mu_{s}$ all even. Here $\mu=\left(\mu_{1}, \ldots, \mu_{s}\right)$. 
Proof. Let $w=x \sigma \in W$ with $x \in(\mathbb{Z} / 2 \mathbb{Z})^{n}, \sigma \in S_{n}$. The conjugacy class of $w$ in $W^{\prime}$ is a single $W$-conjugacy class if and only if $w=v^{-1} w v$ for some $v \in W^{\prime}-W$. This is so if and only if there exist $y=\left(y_{1}, \ldots, y_{n}\right) \in(\mathbb{Z} / 2 \mathbb{Z})^{n}$ and $\tau \in S_{n}$ such that $y_{1} \cdots y_{n}=-1, \tau \sigma=\sigma \tau$, and $y^{-1} \cdot{ }^{\sigma} y=x^{-1} \cdot{ }^{\tau} x$, where ${ }^{\sigma} y$ denotes the action of $\sigma \in S_{n}$ on $y \in(\mathbb{Z} / 2 \mathbb{Z})^{n}$. The lemma can be deduced from this observation.

The conjugacy classes conj $W_{W^{\prime}}(w)$ of $w \in W$ in $W^{\prime}$ are in bijective correspondence with the subset $\mathcal{P}_{n, e}^{(2)}$ of $\mathcal{P}_{n}^{(2)}$ (cf. (20)) given by

$$
\mathcal{P}_{n, e}^{(2)}=\left\{(\mu, \nu) \in \mathcal{P}_{n}^{(2)} \mid l(\nu) \text { is even }\right\} .
$$

Let $\operatorname{conj}_{W^{\prime}}(w)(w \in W)$ correspond to $(\mu, \nu)=\left(\left(\mu_{1}, \ldots, \mu_{s}\right),\left(\nu_{1}, \ldots, \nu_{t}\right)\right)$, with $t$ even. Then, as in the case of symplectic group $\mathrm{Sp}_{2 n}$,

$$
T^{w F} \cong \mathbb{F}_{q^{\mu_{1}}}^{\times} \times \cdots \times \mathbb{F}_{q^{\mu_{s}}}^{\times} \times C_{q^{\nu_{1}}} \times \cdots \times C_{q^{\nu t}},
$$

and

$$
\frac{1}{|T w F|} \sum_{a \in T w F} \lambda(\operatorname{tr} a)=\frac{K\left(\lambda^{\left(\mu_{1}\right)}\right) \cdots K\left(\lambda^{\left(\mu_{s}\right)}\right) K\left(\lambda^{\left(\nu_{1}\right)}\right) \cdots K\left(\lambda^{\left(\nu_{t}\right)}\right)}{\left(q^{\mu_{1}}-1\right) \cdots\left(q^{\mu_{s}}-1\right)\left(q^{\nu_{1}}+1\right) \cdots\left(q^{\nu_{t}}+1\right)} .
$$

It is important to observe that the above sum depends only on the conjugacy classes of $w \in W$ in $W^{\prime}$. In the RHS of the following expression, we group together $w^{\prime}$ s in the same conjugacy classes in $W^{\prime}$.

$$
\sum_{g \in \mathrm{SO}^{+}(2 n, q)} \lambda(\operatorname{tr} g)=\frac{\left|\mathrm{SO}^{+}(2 n, q)\right|}{|W|} \sum_{w \in W} \frac{1}{|T w F|} \sum_{a \in T w F} \lambda(\operatorname{tr} a) .
$$

Then we obtain the next theorem from (21) and the above calculations. Here one must note that

$$
\left|\operatorname{conj}_{W^{\prime}}(w)\right|=\frac{\left|W^{\prime}\right|}{2^{l(\mu)+l(\nu)} z_{\mu} z_{\nu}}=\frac{2|W|}{2^{l(\mu)+l(\nu)} z_{\mu} z_{\nu}} .
$$

Theorem 3.5.

$$
\begin{aligned}
& \sum_{g \in \mathrm{SO}^{+}(2 n, q)} \lambda(\operatorname{tr} g) \\
= & 2 q^{n^{2}-n}\left(q^{n}-1\right) \prod_{j=1}^{n-1}\left(q^{2 j}-1\right) \\
& \times \sum_{(\mu, \nu) \in \mathcal{P}_{n, e}^{(2)}} \frac{K\left(\lambda^{\left(\mu_{1}\right)}\right) \cdots K\left(\lambda^{\left(\mu_{s}\right)}\right) K\left(\lambda^{\left(\nu_{1}\right)}\right) \cdots K\left(\lambda^{\left(\nu_{t}\right)}\right)}{2^{l(\mu)+l(\nu)} z_{\mu} z_{\nu}\left(q^{\mu_{1}}-1\right) \cdots\left(q^{\mu_{s}}-1\right)\left(q^{\nu_{1}}+1\right) \cdots\left(q^{\nu_{t}}+1\right)} .
\end{aligned}
$$




\section{4. $\mathrm{SO}^{-}(2 n, q)$}

Let $J^{-}$be the $2 n \times 2 n$ matrix with $\left[\begin{array}{ll}0 & 1 \\ 1 & 0\end{array}\right]$ along the first $n-1$ diagonal blocks, $\left[\begin{array}{cc}1 & 0 \\ 0 & -\varepsilon\end{array}\right]$ for the last diagonal block and zeros elsewhere, where $\varepsilon$ is a fixed element in $\mathbb{F}_{q}^{\times}-\mathbb{F}_{q}^{\times^{2}}$. Let

$$
G=\mathrm{SO}^{-}\left(2 n, \overline{\mathbb{F}}_{q}\right)=\left\{\left.g \in \mathrm{GL}\left(2 n, \overline{\mathbb{F}}_{q}\right)\right|^{t} g J^{-} g=J^{-}, \operatorname{det} g=1\right\} .
$$

Then $G^{\mathrm{Fr}_{q}}=\mathrm{SO}^{-}(2 n, q)$, where $\operatorname{Fr}_{q}$ is the standard Frobenius map on $G$.

$G$ is a form of $\mathrm{SO}_{2 n}^{+}$over $\mathbb{F}_{q}$. In other words, $G=\mathrm{SO}_{2 n}^{-}$is defined over $\mathbb{F}_{q}$, and is isomorphic to $\mathrm{SO}_{2 n}^{+}$over $\overline{\mathbb{F}}_{q}$. Indeed, an isomorphism $\phi: \mathrm{SO}_{2 n}^{+} \rightarrow \mathrm{SO}_{2 n}^{-}$ defined over $\mathbb{F}_{q^{2}}$ is given by $\phi(g)=A g A^{-1}$, where $A$ is the block diagonal matrix with 1's along the diagonal and the last diagonal block is

$$
2^{-\frac{1}{2}}\left[\begin{array}{cc}
1 & 1 \\
\varepsilon^{-\frac{1}{2}} & -\varepsilon^{-\frac{1}{2}}
\end{array}\right] \text {. }
$$

Hence $G=\mathrm{SO}_{2 n}^{-}$can be identified with $\mathrm{SO}_{2 n}^{+}$equipped with the Frobenius map $F=\phi^{-1} \circ \operatorname{Fr}_{q} \circ \phi$. In particular,

$$
\left|\mathrm{SO}_{2 n}^{+F}\right|=\left|\mathrm{SO}^{-}(2 n, q)\right|=q^{n^{2}-n}\left(q^{n}+1\right) \prod_{j=1}^{n-1}\left(q^{2 j}-1\right) .
$$

We note here that $F$ is the twisted Frobenius map given by

$$
F(g)=A^{-1} \operatorname{Fr}_{q}\left(A g A^{-1}\right) A=B \operatorname{Fr}_{q}(g) B^{-1},
$$

where $B=A^{-1} \operatorname{Fr}_{q}(A)$ is the block diagonal matrix with 1's along the diagonal except $\pm\left[\begin{array}{ll}0 & 1 \\ 1 & 0\end{array}\right]$ for the last $2 \times 2$ diagonal block. Here the sign is plus or minus depending on whether 2 is a square in $\mathbb{F}_{q}^{\times}$or not. So $F=\psi \circ \operatorname{Fr}_{q}$, where

$$
\psi: \mathrm{SO}_{2 n}^{+} \rightarrow \mathrm{SO}_{2 n}^{+} \text {is given by } \psi(g)=B g B^{-1} \text {. }
$$

The subgroup $T$ of diagonal matrices in $\mathrm{SO}_{2 n}^{+}$is again $F$-stable, but $F$ acts nontrivially on the Weyl group $W=N(T) / T$.

Theorem 3.6.

$$
\begin{aligned}
& \quad \sum_{g \in \mathrm{SO}^{-}(2 n, q)} \lambda(\operatorname{trg}) \\
& =-2 q^{n^{2}-n}\left(q^{n}+1\right) \prod_{j=1}^{n-1}\left(q^{2 j}-1\right) \\
& \quad \times \sum_{(\mu, \nu) \in \mathcal{P}_{n, o}^{(2)}} \frac{K\left(\lambda^{\left(\mu_{1}\right)}\right) \cdots K\left(\lambda^{\left(\mu_{s}\right)}\right) K\left(\lambda^{\left(\nu_{1}\right)}\right) \cdots K\left(\lambda^{\left(\nu_{t}\right)}\right)}{2^{l(\mu)+l(\nu)} z_{\mu} z_{\nu}\left(q^{\mu_{1}}-1\right) \cdots\left(q^{\mu_{s}}-1\right)\left(q^{\nu_{1}}+1\right) \cdots\left(q^{\nu_{t}}+1\right)},
\end{aligned}
$$

where $\mathcal{P}_{n, o}^{(2)}$ denotes the subset of $\mathcal{P}_{n}^{(2)}$ given by

$$
\mathcal{P}_{n, o}^{(2)}=\left\{(\mu, \nu) \in \mathcal{P}_{n}^{(2)} \mid l(\nu) \text { is odd }\right\} .
$$


Proof. The action of $\psi$ (cf. (26)) on $T$ is given by $\left.\psi\right|_{T}=w_{0}=(1, \ldots, 1,-1) \in$ $W^{\prime}=(\mathbb{Z} / 2 \mathbb{Z})^{n} \rtimes S_{n}$ if we view $W^{\prime}$ as a group of automorphisms of $T$. Let $w \in W$. Then ${ }_{w} F={ }_{w} w_{0} \operatorname{Fr}_{q}$ on $T$, and hence $T w F=T^{w w_{0}} \mathrm{Fr}_{q}$.

The Gauss sum for $\mathrm{SO}^{-}(2 n, q)$ is

$$
\sum_{g \in \mathrm{SO}^{-}(2 n, q)} \lambda(\operatorname{tr} g)=\frac{\left|\mathrm{SO}^{-}(2 n, q)\right|}{|W|} \sum_{w \in W} \frac{1}{\left|T^{w w_{0}} \mathrm{Fr}_{q}\right|} \sum_{a \in T^{w w_{0}} \mathrm{Fr}_{q}} \lambda(\operatorname{tr} a) .
$$

Observe now that $W w_{0}$ is the subset of odd signed permutations in $(\mathbb{Z} / 2 \mathbb{Z})^{n} \rtimes$ $S_{n}$ and the conjugacy classes conj $W_{W^{\prime}}\left(w w_{0}\right)$ of $w w_{0} \in W w_{0}$ in $W^{\prime}$ are in bijective correspondence with the subset $\mathcal{P}_{n, o}^{(2)}$ in $(27)$ of $\mathcal{P}_{n}^{(2)}$.

As in the case of $\mathrm{SO}_{2 n}^{+}$, in the above summation, the sum corresponding to each $w \in W$ depends only on the conjugacy classes of $w w_{0} \in W w_{0}$ in $W^{\prime}$. The result now follows from (22), (25). Here one must observe that (22) also holds for $w w_{0} \in W w_{0}$, if $\operatorname{conj}_{W^{\prime}}\left(w w_{0}\right)$ corresponds to $(\mu, \nu)=$ $\left(\left(\mu_{1}, \ldots, \mu_{s}\right),\left(\nu_{1}, \ldots, \nu_{t}\right)\right) \in \mathcal{P}_{n, o}^{(2)}$, with $t$ odd. Also, one must note that

$$
\left|\operatorname{conj}_{W^{\prime}}\left(w w_{0}\right)\right|=\frac{\left|W^{\prime}\right|}{2^{l(\mu)+l(\nu)} z_{\mu} z_{\nu}}=\frac{2|W|}{2^{l(\mu)+l(\nu)} z_{\mu} z_{\nu}} .
$$

\section{5. $\mathrm{SO}(2 n+1, q)$}

Let

$$
G=\mathrm{SO}\left(2 n+1, \overline{\mathbb{F}}_{q}\right)=\left\{\left.g \in \mathrm{GL}\left(2 n+1, \overline{\mathbb{F}}_{q}\right)\right|^{t} g J g=J, \operatorname{det} g=1\right\} .
$$

Here $J$ denotes the $(2 n+1) \times(2 n+1)$ matrix

$$
J=\left[\begin{array}{ccc}
0 & 1_{n} & 0 \\
1_{n} & 0 & 0 \\
0 & 0 & 1
\end{array}\right]
$$

$F$ is the standard Frobenius map $\operatorname{Fr}_{q}$ on $G$, and $G^{F}=\operatorname{SO}(2 n+1, q)$, with

$$
|\mathrm{SO}(2 n+1, q)|=|\operatorname{Sp}(2 n, q)|=q^{n^{2}} \prod_{j=1}^{n}\left(q^{2 j}-1\right) .
$$

$T=\{$ diagonal matrices in $G\}=\left\{\operatorname{diag}\left(\alpha_{1}, \ldots, \alpha_{n}, \alpha_{1}^{-1}, \ldots, \alpha_{n}^{-1}, 1\right)\right\}$ is an $F$ stable maximal torus of $G$.

For the rest of this subsection, one is referred to the Section 3.1. As in the symplectic group case, $W=N(T) / T \cong(\mathbb{Z} / 2 \mathbb{Z})^{n} \rtimes S_{n}$ is the Weyl group with respect to $T$, and $F$-conjugacy classes in $W$ coincide with conjugacy classes in $W$.

If $\operatorname{conj}(w)(w \in W)$ corresponds to $(\mu, \nu)=\left(\left(\mu_{1}, \ldots, \mu_{s}\right),\left(\nu_{1}, \ldots, \nu_{t}\right)\right) \in$ $\mathcal{P}_{n}^{(2)}$, then

$$
\begin{aligned}
T^{w} F=\{ & \operatorname{diag}\left(t_{\mu_{1}}\left(\alpha_{1}\right), \ldots, t_{\mu_{s}}\left(\alpha_{s}\right), t_{\nu_{1}}\left(\beta_{1}\right), \ldots, t_{\nu_{t}}\left(\beta_{t}\right),\right. \\
& \left.t_{\mu_{1}}\left(\alpha_{1}^{-1}\right), \ldots, t_{\mu_{s}}\left(\alpha_{s}^{-1}\right), t_{\nu_{1}}\left(\beta_{1}^{-1}\right), \ldots, t_{\nu_{t}}\left(\beta_{t}^{-1}\right), 1\right)
\end{aligned}
$$




$$
\left.\alpha_{1} \in \mathbb{F}_{q^{\mu_{1}}}^{\times}, \ldots, \alpha_{s} \in \mathbb{F}_{q^{\mu_{s}}}^{\times}, \beta_{1} \in C_{q^{\nu_{1}}}, \ldots, \beta_{t} \in C_{q^{\nu_{t}}}\right\} .
$$

So

$$
\sum_{a \in T w_{F}} \lambda(\operatorname{tr} a)=\lambda(1)(-1)^{t} K\left(\lambda^{\left(\mu_{1}\right)}\right) \cdots K\left(\lambda^{\left(\mu_{s}\right)}\right) K\left(\lambda^{\left(\nu_{1}\right)}\right) \cdots K\left(\lambda^{\left(\nu_{t}\right)}\right),
$$

which is $\lambda(1)$ times the expression in (22).

Now, from (1), (21) and the above, the next theorem follows.

Theorem 3.7.

$$
\begin{aligned}
& \quad \sum_{g \in \mathrm{SO}(2 n+1, q)} \lambda(\operatorname{tr} g)=\lambda(1) \sum_{g \in S p(2 n, q)} \lambda(\operatorname{tr} g) \\
& =\lambda(1) q^{n^{2}} \prod_{j=1}^{n}\left(q^{2 j}-1\right) \\
& \quad \times \sum_{(\mu, \nu) \in \mathcal{P}_{n}^{(2)}} \frac{(-1)^{l(\nu)} K\left(\lambda^{\left(\mu_{1}\right)}\right) \cdots K\left(\lambda^{\left(\mu_{s}\right)}\right) K\left(\lambda^{\left(\nu_{1}\right)}\right) \cdots K\left(\lambda^{\left(\nu_{t}\right)}\right)}{2^{l(\mu)+l(\nu)} z_{\mu} z_{\nu}\left(q^{\mu_{1}}-1\right) \cdots\left(q^{\mu_{s}}-1\right)\left(q^{\nu_{1}}+1\right) \cdots\left(q^{\nu_{t}}+1\right)} .
\end{aligned}
$$

Remark 3.8. The above relation was already observed in [11, (5.12)]. However, thanks to the main formula (1), the present argument gives more 'philosophical' reason that this should be so.

\section{Identities}

On the one hand, the expressions of Gauss sums in the last section were obtained by applying the main formula (1) to various finite classical groups. On the other hand, alternative expressions of those sums were gotten by using Bruhat decompositions with respect to certain maximal parabolic subgroups of the finite classical groups (cf. $[7,8,9,10,11,12,15])$. In this section, equating two different expressions of the Gauss sum for each finite classical group, we will be able to produce some new identities. by

For $\gamma \in \mathbb{F}_{q}^{\times}$, we have defined the Kloosterman sum for $G L(t, q)$ in $[10,(4.3)]$

$$
\begin{gathered}
\left.K_{\mathrm{GL}(t, q)}(\lambda ; \gamma)=\sum_{\substack{g \in \mathrm{GL}(t, q) \\
K_{\mathrm{GL}(0, q)}} \lambda(\operatorname{tr} g+\gamma)=1,} \operatorname{tr}^{-1}\right)(t \geqslant 1), \\
K_{\mathrm{GL}(t, q)}(\lambda)=K_{\mathrm{GL}(t, q)}(\lambda ; 1) .
\end{gathered}
$$

The expressions of the Gauss sum for $\operatorname{Sp}(2 n, q)$ in Theorem 3.1 and the sentence following $[10,(5.13)]$ yield the next theorem.

Theorem 4.1.

$$
\sum_{(\mu, \nu) \in \mathcal{P}_{n}^{(2)}} \frac{(-1)^{l(\nu)} K\left(\lambda^{\left(\mu_{1}\right)}\right) \cdots K\left(\lambda^{\left(\mu_{s}\right)}\right) K\left(\lambda^{\left(\nu_{1}\right)}\right) \cdots K\left(\lambda^{\left(\nu_{t}\right)}\right)}{2^{l(\mu)+l(\nu)} z_{\mu} z_{\nu}\left(q^{\mu_{1}}-1\right) \cdots\left(q^{\mu_{s}}-1\right)\left(q^{\nu_{1}}+1\right) \cdots\left(q^{\nu_{t}}+1\right)}
$$




$$
=q^{-\left(\begin{array}{c}
n \\
2
\end{array}\right)} \prod_{j=1}^{n}\left(q^{2 j}-1\right)^{-1} \times \sum_{\substack{0 \leqslant r \leqslant n \\
r \text { even }}} q^{r n-r^{2} / 4}\left[\begin{array}{l}
n \\
r
\end{array}\right] \prod_{q}^{r / 2}\left(q^{2 j-1}-1\right) K_{\mathrm{GL}(n-r, q)}(\lambda)
$$

where $\mathcal{P}_{n}^{(2)}, z_{\mu}$ are respectively as in (20), (18).

Remark 4.2. In [10, Lemma 4.2], it is shown that the Kloosterman sums for general linear groups (28) satisfy the recursive relation

$$
\begin{aligned}
K_{\mathrm{GL}(t, q)}(\lambda ; \gamma)= & q^{t-1} K_{\mathrm{GL}(t-1, q)}(\lambda ; \gamma) K(\lambda ; \gamma) \\
& +q^{2 t-2}\left(q^{t-1}-1\right) K_{\mathrm{GL}(t-2, q)}(\lambda ; \gamma)(t \geqslant 2) .
\end{aligned}
$$

Here $K(\lambda ; \gamma)=K_{\mathrm{GL}(1, q)}(\lambda ; \gamma)$ is the usual Kloosterman sum as in (4) with $m=1$.

From this relation, for $t \geqslant 1$ one can show

$$
\begin{aligned}
K_{\mathrm{GL}(t, q)}(\lambda ; \gamma)= & q^{\frac{1}{2}(t-2)(t+1)} \\
& \times \sum_{l=1}^{[(t+2) / 2]} q^{l} K(\lambda ; \gamma)^{t+2-2 l} \sum_{j_{1}, \ldots, j_{l-1}} \prod_{\nu=1}^{l-1}\left(q^{j_{\nu}-2 \nu}-1\right),
\end{aligned}
$$

where the second sum is over all integers $j_{1}, \ldots, j_{l-1}$ satisfying

$$
2 l-1 \leqslant j_{l-1} \leqslant j_{l-2} \leqslant \cdots \leqslant j_{1} \leqslant t+1,
$$

and we agree that it is 1 for $l=1$.

A 'twisted average' version of Theorem 4.1 is obtained from the expressions of the Gauss sum for $\operatorname{GSp}(2 n, q)$ in Theorem 3.2 and [10, (5.8)].

\section{Theorem 4.3.}

$$
\begin{aligned}
& \sum_{(\mu, \nu) \in \mathcal{P}_{n}^{(2)}} \frac{(-1)^{l(\nu)} \sum_{\gamma \in \mathbb{F}_{q}^{\times}} \chi^{n}(\gamma) K\left(\lambda^{\left(\mu_{1}\right)} ; \gamma\right) \cdots K\left(\lambda^{\left(\mu_{s}\right)} ; \gamma\right) K\left(\lambda^{\left(\nu_{1}\right)} ; \gamma\right) \cdots K\left(\lambda^{\left(\nu_{t}\right)} ; \gamma\right)}{2^{l(\mu)+l(\nu)} z_{\mu} z_{\nu}\left(q^{\mu_{1}}-1\right) \cdots\left(q^{\mu_{s}}-1\right)\left(q^{\nu_{1}}+1\right) \cdots\left(q^{\nu^{t}}+1\right)} \\
= & q^{-\left(\begin{array}{c}
n \\
2
\end{array}\right)} \prod_{j=1}^{n}\left(q^{2 j}-1\right)^{-1} \\
& \times \sum_{\substack{0 \leqslant r \leqslant n \\
r \text { even }}} q^{r n-r^{2} / 4}\left[\begin{array}{l}
n \\
r
\end{array}\right]_{q} \prod_{j=1}^{r / 2}\left(q^{2 j-1}-1\right) \sum_{\gamma \in \mathbb{F}_{q}^{\times}} \chi^{n}(\gamma) K_{\mathrm{GL}(t, q)}(\lambda ; \gamma),
\end{aligned}
$$

where $\mathcal{P}_{n}^{(2)}, z_{\mu}, K_{G L(t, q)}(\lambda ; \gamma)$ are respectively as in (20), (18), (28).

Remark 4.4. The twisted average Kloosterman sum $\sum_{\gamma \in \mathbb{F}_{q}^{\times}} \eta(\gamma) K_{\mathrm{GL}(t, q)}(\lambda ; \gamma)$ for $\operatorname{GL}(t, q)$ satisfies similar relation as in (29) (cf. [10, (5.9)]). Here $\eta$ is any multiplicative character of $\mathbb{F}_{q}$. From that relation, one can find an explicit expression for the sum (cf. (30)). 


$$
\begin{aligned}
& \sum_{\gamma \in \mathbb{F}_{q}^{\times}} \eta(\gamma) K_{\mathrm{GL}(t, q)}(\lambda ; \gamma) \\
= & q^{\frac{1}{2}(t-2)(t+1)} \sum_{l=1}^{[(t+2) / 2]} q^{l} \sum_{\gamma} \eta(\gamma) K(\lambda ; \gamma)^{t+2-2 l} \sum_{j_{1}, \ldots, j_{l-1}} \prod_{\nu=1}^{l-1}\left(q^{j_{\nu}-2 \nu}-1\right),
\end{aligned}
$$

where $\gamma$ in the second sum is over $\mathbb{F}_{q}^{\times}$, the third sum runs over the same set of integers as in (31), and we understand that it is 1 for $l=1$.

In the remark following [15, Theorem 4.3], it is shown that

$$
\begin{gathered}
\sum_{g \in \mathrm{SO}^{+}(2 n, q)} \lambda(\operatorname{tr} g)=q^{-n} \sum_{g \in \operatorname{Sp}(2 n, q)} \lambda(\operatorname{tr} g) \\
=q^{\left(\begin{array}{c}
n \\
2
\end{array}\right)} \sum_{\substack{0 \leqslant r \leqslant n \\
r \text { even }}} q^{r n-r^{2} / 4}\left[\begin{array}{c}
n \\
r
\end{array}\right] \prod_{q}^{r / 2}\left(q^{2 j-1}-1\right) K_{\mathrm{GL}(n-r, q)}(\lambda) .
\end{gathered}
$$

So we get the following identities, in view of (32), from Theorems 3.1, 3.5, and 4.1 .

\section{Theorem 4.5.}

$$
\begin{aligned}
& \sum_{(\mu, \nu) \in \mathcal{P}_{n, e}^{(2)}} \frac{K\left(\lambda^{\left(\mu_{1}\right)}\right) \cdots K\left(\lambda^{\left(\mu_{s}\right)}\right) K\left(\lambda^{\left(\nu_{1}\right)}\right) \cdots K\left(\lambda^{\left(\nu_{t}\right)}\right)}{2^{l(\mu)+l(\nu)} z_{\mu} z_{\nu}\left(q^{\mu_{1}}-1\right) \cdots\left(q^{\mu_{s}}-1\right)\left(q^{\nu_{1}}+1\right) \cdots\left(q^{\nu_{t}}+1\right)} \\
= & \frac{1}{2}\left(q^{n}+1\right) \sum_{\substack{(\mu, \nu) \in \mathcal{P}_{n}^{(2)} \\
2^{l(\mu)+l(\nu)} z_{\mu} z_{\nu}\left(q^{\mu_{1}}-1\right) \cdots\left(q^{\mu_{s}}-1\right)\left(q^{\nu_{1}}+1\right) \cdots\left(q^{\nu_{t}}+1\right)}} \frac{(-1)^{l(\nu)} K\left(\lambda^{\left(\mu_{1}\right)}\right) \cdots K\left(\lambda^{\left(\mu_{s}\right)}\right) K\left(\lambda^{\left(\nu_{1}\right)}\right) \cdots K\left(\lambda^{\left(\nu_{t}\right)}\right)}{=^{n}} \frac{1}{2}\left(q^{n}+1\right) q^{-\left(\begin{array}{c}
n \\
2
\end{array}\right)} \prod_{j=1}^{n}\left(q^{2 j}-1\right)^{-1} \sum_{\substack{0 \leqslant r \leqslant n \\
r \text { even }}} q^{r n-r^{2} / 4}\left[\begin{array}{c}
n \\
r
\end{array}\right] \prod_{q}^{r / 2}\left(q^{2 j-1}-1\right) K_{\mathrm{GL}(n-r, q)}(\lambda),
\end{aligned}
$$

where $\mathcal{P}_{n}^{(2)}, \mathcal{P}_{n, e}^{(2)}, z_{\mu}, K_{\mathrm{GL}(t, q)}(\lambda)$ are respectively as in (20), (24), (18), (28).

Put $\Sigma, \Sigma_{\text {even }}$ and $\Sigma_{\text {odd }}$ to be the summation of the following terms over $(\mu, \nu)$ in $\mathcal{P}_{n}^{(2)}, \mathcal{P}_{n, e}^{(2)}$ and $\mathcal{P}_{n, o}^{(2)}$, respectively.

$$
\frac{(-1)^{l(\nu)} K\left(\lambda^{\left(\mu_{1}\right)}\right) \cdots K\left(\lambda^{\left(\mu_{s}\right)}\right) K\left(\lambda^{\left(\nu_{1}\right)}\right) \cdots K\left(\lambda^{\left(\nu_{t}\right)}\right)}{2^{l(\mu)+l(\nu)} z_{\mu} z_{\nu}\left(q^{\mu_{1}}-1\right) \cdots\left(q^{\mu_{s}}-1\right)\left(q^{\nu_{1}}+1\right) \cdots\left(q^{\nu_{t}}+1\right)}
$$

(cf. (27) for $\mathcal{P}_{n, o}^{(2)}$ ).

As $\mathcal{P}_{n}^{(2)}=\mathcal{P}_{n, e}^{(2)} \cup \mathcal{P}_{n, o}^{(2)}$ is a disjoint union, $\sum=\sum_{\text {even }}+\sum_{\text {odd }}$ by definition. As we saw in Theorem 4.5,

$$
\sum_{\text {even }}=\frac{1}{2}\left(q^{n}+1\right) \sum
$$


Hence, we obtain

$$
\sum_{\text {odd }}=\frac{1}{2}\left(1-q^{n}\right) \sum .
$$

These results are summarized in the following corollary (cf. Theorem 4.1).

Corollary 4.6. (a)

$$
\begin{aligned}
& \sum_{(\mu, \nu) \in \mathcal{P}_{n, o}^{(2)}} \frac{K\left(\lambda^{\left(\mu_{1}\right)}\right) \cdots K\left(\lambda^{\left(\mu_{s}\right)}\right) K\left(\lambda^{\left(\nu_{1}\right)}\right) \cdots K\left(\lambda^{\left(\nu_{t}\right)}\right)}{2^{l(\mu)+l(\nu)} z_{\mu} z_{\nu}\left(q^{\mu_{1}}-1\right) \cdots\left(q^{\mu_{s}}-1\right)\left(q^{\nu_{1}}+1\right) \cdots\left(q^{\nu_{t}}+1\right)} \\
= & \frac{1}{2}\left(q^{n}-1\right) \sum_{(\mu, \nu) \in \mathcal{P}_{n}^{(2)}} \frac{(-1)^{l(\nu)} K\left(\lambda^{\left(\mu_{1}\right)}\right) \cdots K\left(\lambda^{\left(\mu_{s}\right)}\right) K\left(\lambda^{\left(\nu_{1}\right)}\right) \cdots K\left(\lambda^{\left(\nu_{t}\right)}\right)}{2^{l(\mu)+l(\nu)} z_{\mu} z_{\nu}\left(q^{\mu_{1}}-1\right) \cdots\left(q^{\mu_{s}}-1\right)\left(q^{\nu_{1}}+1\right) \cdots\left(q^{\nu_{t}}+1\right)} \\
= & \frac{1}{2}\left(q^{n}-1\right) q^{-\left(\begin{array}{c}
n \\
2
\end{array}\right)} \prod_{j=1}^{n}\left(q^{2 j}-1\right)^{-1} \sum_{\substack{0 \leqslant r \leqslant n \\
r \text { even }}} q^{r n-r^{2} / 4}\left[\begin{array}{c}
n \\
r
\end{array}\right] \prod_{q}^{r / 2}\left(q^{2 j-1}-1\right) K_{\mathrm{GL}(n-r, q)}(\lambda) .
\end{aligned}
$$

$$
\begin{aligned}
& \left(q^{n}+1\right)^{-1} \sum_{(\mu, \nu) \in \mathcal{P}_{n, e}^{(2)}} \frac{K\left(\lambda^{\left(\mu_{1}\right)}\right) \cdots K\left(\lambda^{\left(\mu_{s}\right)}\right) K\left(\lambda^{\left(\nu_{1}\right)}\right) \cdots K\left(\lambda^{\left(\nu_{t}\right)}\right)}{2^{l(\mu)+l(\nu)} z_{\mu} z_{\nu}\left(q^{\mu_{1}}-1\right) \cdots\left(q^{\mu_{s}}-1\right)\left(q^{\nu_{1}}+1\right) \cdots\left(q^{\nu_{t}}+1\right)} \\
= & \left(q^{n}-1\right)^{-1} \sum_{(\mu, \nu) \in \mathcal{P}_{n, o}^{(2)}} \frac{K\left(\lambda^{\left(\mu_{1}\right)}\right) \cdots K\left(\lambda^{\left(\mu_{s}\right)}\right) K\left(\lambda^{\left(\nu_{1}\right)}\right) \cdots K\left(\lambda^{\left(\nu_{t}\right)}\right)}{2^{l(\mu)+l(\nu)} z_{\mu} z_{\nu}\left(q^{\mu_{1}}-1\right) \cdots\left(q^{\mu_{s}}-1\right)\left(q^{\nu_{1}}+1\right) \cdots\left(q^{\nu_{t}}+1\right)} .
\end{aligned}
$$

Remark 4.7. It would be interesting if one could obtain the above identities purely in combinatorial manner.

From Theorems 3.5, 3.6 and the last corollary we get the following somewhat suprising identities:

$$
\begin{aligned}
\sum_{g \in \mathrm{SO}^{-}(2 n, q)} \lambda(\operatorname{tr} g) & =2 q^{n^{2}-n}\left(q^{n}+1\right) \prod_{j=1}^{n-1}\left(q^{2 j}-1\right) \sum_{\text {odd }} \\
= & -2 q^{n^{2}-n}\left(q^{n}-1\right) \prod_{j=1}^{n-1}\left(q^{2 j}-1\right) \sum_{\text {even }} \\
= & -\sum_{g \in \mathrm{SO}^{+}(2 n, q)} \lambda(\operatorname{tr} g) .
\end{aligned}
$$

Now, we have enough information to have complete relations between Gauss sums for various special orthogonal groups and symplectic group. (cf. Theorem 3.7, (32), (34))

Theorem 4.8. (a) $\sum_{g \in \operatorname{SO}(2 n+1, q)} \lambda(\operatorname{tr} g)=\lambda(1) \sum_{g \in \operatorname{Sp}(2 n, q)} \lambda(\operatorname{tr} g)$, 
(b) $\sum_{g \in \mathrm{SO}^{+}(2 n, q)} \lambda(\operatorname{tr} g)=q^{-n} \sum_{g \in \operatorname{Sp}(2 n, q)} \lambda(\operatorname{tr} g)$,

(c) $\sum_{g \in \mathrm{SO}^{-}(2 n, q)} \lambda(\operatorname{tr} g)=-q^{-n} \sum_{g \in \operatorname{Sp}(2 n, q)} \lambda(\operatorname{tr} g)=-\sum_{g \in \mathrm{SO}^{+}(2 n, q)} \lambda(\operatorname{tr} g)$.

On the one hand, $\sum_{g \in \mathrm{SO}^{-}(2 n, q)} \lambda(\operatorname{tr} g)$ equals the negative of the expression in (33). On the other hand, it equals the expression in $[8,(5.10)]$ (cf. [8, (4.13)], $[13,(3.1)])$. Thus the following identity holds, which also can be proved directly for example using induction on $r$.

\section{Proposition 4.9.}

$$
\begin{aligned}
& K(\lambda) \sum_{\substack{0 \leqslant r \leqslant n-1 \\
r \text { even }}} q^{n r-r^{2} / 4}\left[\begin{array}{c}
n-1 \\
r
\end{array}\right] \prod_{q}^{r / 2}\left(q^{2 j-1}-1\right) K_{\mathrm{GL}(n-1-r, q)}(\lambda) \\
& +(q+1) \sum_{\substack{0 \leqslant r \leqslant n-1 \\
r \text { odd }}} q^{n r-(r+1)^{2} / 4}\left[\begin{array}{c}
n-1 \\
r
\end{array}\right]_{q} \prod_{j=1}^{(r+1) / 2}\left(q^{2 j-1}-1\right) K_{\mathrm{GL}(n-1-r, q)}(\lambda) \\
& =q^{1-n} \sum_{\substack{0 \leqslant r \leqslant n \\
r \text { even }}}\left[\begin{array}{c}
n \\
r
\end{array}\right]_{q} q^{n r-r^{2} / 4} \prod_{j=1}^{r / 2}\left(q^{2 j-1}-1\right) K_{\mathrm{GL}(n-r, q)}(\lambda) .
\end{aligned}
$$

\section{References}

[1] G. E. Andrews, The Theory of Partitions, Cambridge University Press, Cambridge, 1998.

[2] B. C. Berndt, R. J. Evans, and K. S. Williams, Gauss and Jacobi Sums, Canad. Math. Soc. Ser. Monographs Adv. Texts 21, Wiley, New York, 1998.

[3] H. Chae and D. S. Kim, Identities arising from Gauss sums for finite classical groups, J. Number Theory 128 (2008), no. 7, 2010-2024.

[4] _ A generalization of power moments of Kloosterman sums, Arch. Math. (Basel) 89 (2007), no. 2, 152-156.

[5] P. Deligne, Applications de la formula des traces aux sommes trigonométriques, in Cohomologie Etale, Séminaire de Géométrie Algébrique du Bois-Marie SGA 4 1/2 by P. Deligne, Lecture Notes in Math. 569 (1977), 168-232.

[6] Y.-K. Jeong, I.-S. Lee, H. Oh, and K.-H. Park, Gauss sum for the adjoint representation of $\mathrm{GL}_{n}(q)$ and $\mathrm{SL}_{n}(q)$, Acta Arith. 95 (2000), no. 1, 1-16.

[7] D. S. Kim, Gauss sums for general and special linear groups over a finite field, Arch. Math. (Basel) 69 (1997), no. 4, 297-304.

[8] _ Gauss sums for $\mathrm{O}^{-}(2 n, q)$, Acta Arith. 80 (1997), no. 4, 343-365.

$[9]$ _ Gauss sums for $\mathrm{U}\left(2 n+1, q^{2}\right)$ J. Korean Math. Soc. 34 (1997), no. 4, 871-894.

[10] _ Gauss sums for symplectic groups over a finite field, Monatsh. Math. 126 (1998), no. $1,55-71$

[11] _ Gauss sums for $\mathrm{O}(2 n+1, q)$, Finite Fields Appl. 4 (1998), no. 1, 62-86.

$[12]$ _ Gauss sums for $U\left(2 n, q^{2}\right)$, Glasgow Math. J. 40 (1998), no. 1, 79-95.

[13] Exponential sums for $\mathrm{O}^{-}(2 n, q)$ and their applications, Acta Arith. 97 (2001), no. $1,67-86$.

[14] _ Sums for $U\left(2 n, q^{2}\right)$ and their applications, Acta Arith. 101 (2002), no. 4, 339363. 
[15] D. S. Kim and I.-S. Lee, Gauss sums for $\mathrm{O}^{+}(2 n, q)$, Acta Arith. 78 (1996), no. 1, 75-89.

[16] I.-S. Lee and K.-H. Park, Gauss sums for $G_{2}(q)$, Bull. Korean Math. Soc. 34 (1997), no. $2,305-315$.

[17] R. Lidl and H. Niederreiter, Finite Fields, Encyclopedia of Math. Appl. 20, Cambridge University Press, Cambridge, 1987.

[18] I. G. Macdonald, Symmetric Functions and Hall Polynomials, 2nd ed., Oxford Mathematical Monographs, Clarendon Press, Oxford, 1998.

Hi-JoOn Chae

Department of Mathematics EduCATion

HONGIK UNIVERSITY

SEOUl 121-791, Korea

E-mail address: hchae@hongik.ac.kr

Dae SAN Kim

Department of Mathematics

SOGANG University

Seoul 121-742, Korea

E-mail address: dskim@sogang.ac.kr 